\title{
Editor's Perspective on 1
}

Dual-isotope single-photon emission computerized tomography used for prediction of histology and survival in patients after high-dose radiotherapy for malignant astrocytoma. Richard B. Schwartz, B. Leonard Holman, Basem M. Garada, Paulo A. Carvalho, Rebecca Folkerth, Marc S. Schwartz, Jay S. Loeffler, Dennis C. Shrieve, Joseph F. Polak, Peter McL. Black, and Eben Alexander, III

This paper provides an overview of a technique with the potential for predicting accurately the histology of enhancing lesions when there is a question about whether the lesion is radiation-induced necrosis, a recurrent tumor, or may consist of both entities. The complementary imaging techniques take advantage of two of the characteristics of intracranial tumors, namely, blood-brain barrier alterations and changes in focal cerebral flow. While the use of thallium scans is not new, the addition of the HMPAO scans increases the reliability of these techniques. If further experience confirms the authors' findings, this technique will help decrease both the expense and risk of evaluating patients with recurrent glial tumors. 